\title{
Temperamento e Caráter na Resposta à Terapia Cognitivo-Comportamental para Transtorno de Pânico
}

\author{
Débora Cassiane Finkler \\ Hospital de Clínicas de Porto Alegre Brasil \\ Ana Cristina Wesner \\ Elizeth Heldt ${ }^{1}$ \\ Universidade Federal do Rio Grande do Sul
}

\begin{abstract}
RESUMO - O estudo investigou o impacto das características de temperamento e caráter na resposta à terapia cognitivocomportamental (TCC) para pacientes com transtorno de pânico (TP). Um total de 55 pacientes realizou 12 sessões de TCC em grupo (TCCG). A gravidade dos sintomas foi verificada antes e após a intervenção, e o Inventário de Temperamento e Caráter de Cloninger foi aplicado no início do tratamento. Observou-se uma redução significativa na gravidade do TP após a intervenção. Análises de regressão indicaram que as características de temperamento (persistência) e caráter (autodirecionamento e cooperatividade) não se mostraram significativamente relacionadas à melhora da agorafobia e de sintomas depressivos, permanecendo a gravidade inicial desses sintomas como fatores independentes de resposta à TCCG.
\end{abstract}

Palavras-chave: distúrbio do pânico, caráter, personalidade, terapia cognitivo-comportamental, técnicas psicoterapêuticas

\section{Temperament and Character in Response to Cognitive-Behavioral Therapy for Panic Disorder}

\begin{abstract}
This study investigated the impact of temperament and character traits on the response to cognitive-behavioral therapy (CBT) of patients with panic disorder (PD). A total of 55 patients underwent 12 sessions of CBT in group (CBGT). Symptom severity was assessed before and after the intervention and the Cloninger's Temperament and Character Inventory was applied before CBGT. A significant reduction in the severity of the PD severity of the patients after the intervention was observed. Regression analysis indicated that characteristics of temperament (persistence) and character (self-directedness and cooperativeness) were not significantly related to the improvement of agoraphobia and depression, leaving the initial severity of these symptoms as independent factors of response to CBGT.
\end{abstract}

Keywords: panic disorder, character, personality, cognitive-behavioral therapy, psychotherapeutic techniques

A personalidade como um fator de interferência na resposta a diferentes formas de tratamento para os transtornos mentais tem sido foco de pesquisas (Bulik, Sullivan, Carter, McIntosh, \& Joyce, 1999; Corchs et al., 2008; Joyce et al., 2007; Marchesi, Cantoni, Fonto, Giannelli, \& Maggini, 2006; Prasko et al., 2005), considerando estado e traço mensurados por instrumentos específicos de acordo com o constructo (Bienvenu et al., 2009; Kipper et al., 2009; Marchesi, De Panfilis, Cantoni, Giannelli, \& Maggini, 2008). Os resultados dos estudos sugerem que a presença de transtornos da personalidade é preditora da cronicidade de quadros de ansiedade (Skodol, Geier, Grant, \& Hasin, 2014) e mostram-se controversos quanto à resposta ao tratamento (Latas \& Milovanovic, 2014). Especificamente no Transtorno de Pânico (TP), são escassos os estudos que avaliaram a relação entre o efeito das características de personalidade e a resposta ao tratamento de acordo com a conceitualização em traços de temperamento e caráter (Mendoza et al., 2011; Bienvenu et al., 2009).

Estudos têm evidenciado a eficácia de terapia farmacológica (Pollack, 2005) e da Terapia Cognitivo-

1 Endereço para correspondência: Profa Dra Elizeth Heldt, Rua São Manoel, 963, Bairro Rio Branco, Porto Alegre, Brasil. CEP: 90620-110.

E-mail: eliz.h@globo.com
Comportamental (TCC) para o TP (Otto \& Deveney, 2005) com boa resposta a curto (Heldt et al., 2003) e a longo prazo (Heldt et al., 2006), sobretudo para os sintomas residuais. Contudo, alguns pacientes ainda são refratários a diferentes modalidades de tratamentos e suas combinações (Mitte, 2005). Entre os preditores de pior resposta ao tratamento, os estudos relatam a presença de comorbidades com o TP, como a fobia social e o estresse pós-traumático (Chavira et al., 2009), a distimia e a ansiedade generalizada (Heldt et al., 2006). Também a sensibilidade à ansiedade após tratamento farmacológico combinado com TCC individual (Chavira et al., 2009) e os eventos estressores negativos no período de dois anos após TCCG foram preditores de pior resposta ao tratamento (Heldt et al., 2011).

A associação entre as características de temperamento e caráter com transtornos de ansiedade e de personalidade foi estabelecida em estudos prévios. Por exemplo, maiores escores de esquiva ao dano foram preditores de ansiedade generalizada em crianças e adultos (Rettew, Doyle, Kwan, Stanger, \& Hudziak, 2006). Em outro estudo, com 104 pacientes, os resultados indicaram que a gravidade do TP e os traços de autotranscendência são fatores de risco independentes para transtorno de despersonalização (Mendoza et al., 2011). Em relação à personalidade, um estudo encontrou que determinados traços de personalidade, 
como evitativa e dependente, predispõem ao TP e à agorafobia (Bienvenu et al., 2009).

Para avaliar a personalidade, o presente estudo adotou um modelo dimensional, o qual permite acessar as características da personalidade - não definindo psicopatologia ou comorbidades de personalidade, como ocorre no modelo categorial utilizado em manuais diagnósticos. De acordo com Cloninger, a personalidade é composta por fatores de temperamento e caráter que podem revelar suscetibilidade ou proteção a determinadas condições clínicas e a transtornos de personalidade (Cloninger, Svrakic, \& Przybeck, 1993; Fuentes, Tavares, Camargo, \& Gorenstein, 2000). O temperamento compreende dimensões da personalidade relacionadas à tendência hereditária. $\mathrm{O}$ caráter, por sua vez, consiste em aspectos da personalidade referentes à identidade e a autoconceitos moldados ao longo do desenvolvimento e provenientes da aprendizagem em diferentes contextos (Cloninger et al., 1993).

Os fatores de temperamento incluem (a) busca de novidades, que identifica a ativação e iniciação de comportamentos por estímulos novos e a suscetibilidade comportamental à estimulação ambiental; (b) esquiva ao dano, que corresponde à tendência de inibir ou cessar comportamentos perante sinais de estímulos aversivos a fim de evitar punição; (c) dependência de gratificação, que remete à maneira intensa de responder a sinais de recompensa, expressando a dependência da aprovação dos outros; e (d) persistência, relacionada ao fato de insistir em responder de determinada forma, a despeito de reforços intermitentes (Cloninger et al., 1993).

O caráter inclui o autodirecionamento, que representa (a) o reconhecimento de si como um indivíduo autônomo; (b) a cooperatividade, que representa a identificação de si mesmo como uma parte integrante da sociedade e revela a presença de ética, tolerância e ajustamento nas relações interpessoais; (c) a autotranscendência, que indica a identificação de si como parte integrante de todas as coisas, de um todo interdependente, mantendo relação com a imaginação, a capacidade criativa, a religiosidade e o pensamento mágico (Cloninger et al., 1993).

Ainda há controvérsia quanto ao papel das características de temperamento e caráter na resposta a TCC, especialmente no TP. Embora um estudo aponte que características de personalidade influenciam negativamente os resultados do tratamento combinado de fármaco e TCC para TP (Prasko et al., 2005), em outro estudo, a gravidade dos sintomas do TP foi preditor de pior resposta à TCCG, minimizando o impacto de diferentes transtornos de personalidade para esse desfecho (Telch, Kamphuis, \& Schmidt, 2011). Quanto à resposta a tratamento medicamentoso, pacientes sem remissão apresentaram baixos escores em autodirecionamento (Marchesi et al., 2006). Em outros transtornos mentais, os traços de temperamento e de caráter apresentaram diferentes respostas quanto ao tratamento com TCC. No tratamento da bulimia nervosa, altos escores em autodirecionamento foram preditores independentes de melhora rápida e continuada (Bulik et al., 1999). No transtorno obsessivo-compulsivo, menores escores de autodirecionamento estiveram associados com pior resposta à TCC (Corchs et al., 2008). Já no tratamento de depressão, não foi identificada interferência de traços de temperamento e caráter (Joyce et al., 2007). Não foram encontrados estudos que investiguem TCCG para TP e características de temperamento e caráter.

Portanto, observa-se que o impacto das características de temperamento e caráter na resposta ao tratamento do TP permanece uma questão em aberto. A hipótese do estudo é que características de temperamento (maior esquiva ao dano) e de caráter (menor autodirecionamento e cooperatividade) interferem negativamente nos resultados da TCCG de pacientes com TP. Nesse contexto, o objetivo foi avaliar a relação entre as características de temperamento e caráter de pacientes com TP e a resposta à TCCG.

\section{Método}

\section{Delineamento e Participantes}

O estudo consiste de um ensaio clínico não controlado de 12 sessões de TCCG para pacientes com TP. A pesquisa foi realizada de 2006 a 2011, no ambulatório do Hospital de Clínicas de Porto Alegre (HCPA), e os participantes foram encaminhados pelo Programa dos Transtornos de Ansiedade (PROTAN), compondo os grupos para realização a cada semestre, conforme a demanda de encaminhamento e atendendo aos critérios de inclusão. $\mathrm{O}$ estudo foi aprovado pelo Comitê de Ética em Pesquisa do HCPA ( $n^{\circ}$ 07-241). Todos os pacientes assinaram o termo de consentimento livre e esclarecido.

Os critérios de inclusão para participar do estudo foram os seguintes: idade entre 18 e 65 anos, com diagnóstico para TP com ou sem agorafobia, em dose estável de medicação há pelo menos quatro meses e com sintomas residuais de ansiedade antecipatória e evitações fóbicas (avaliados pela Escala de Impressão Clínica Global - ICG >3). O acesso ao grupo foi realizado através de entrevista clínica semiestruturada (Mini International Neuropsychiatric Interview - MINI) para confirmação diagnóstica e avaliação da presença de comorbidades (Amorim, 2000). Foram excluídos os pacientes com sintomas psicóticos, doença clínica significativa ou doença crônica incapacitante nos últimos seis meses da data de avaliação. Um total de 63 pacientes preencheu os critérios de inclusão e iniciou a TCCG.

\section{Instrumentos}

No transcurso do mês antecedente ao início de cada grupo, os dados sociodemográficos e clínicos eram coletados, conforme o protocolo de pesquisa, em entrevista individual realizada por avaliadores treinados (residentes de psiquiatria e psicólogos). A gravidade dos sintomas do TP foi avaliada com os instrumentos Inventário do Pânico, HamiltonAnsiedade (HAM-A) (Hamilton, 1959) e Impressão Clínica Global (ICG) (Guy, 1979), os quais foram aplicados antes e depois da intervenção de TCCG por avaliadores que não participaram da intervenção.

O ICG determina a gravidade global da doença, considerando-se a frequência e a intensidade dos ataques de 
pânico, o grau de ansiedade antecipatória, o nível de evitação fóbica, o prejuízo na qualidade de vida e a necessidade de adequação do tratamento (Guy, 1979). Seu escore varia de 1 (normal, não doente) a 7 (extremamente doente). O Inventário do Pânico caracteriza os ataques de pânico (espontâneo ou situacional, completo ou incompleto), intensidade, duração, frequência, gravidade da agorafobia e ansiedade antecipatória (Heldt et al., 2003). A HAM-A avalia a ansiedade geral com escores que variam de ausente $(0)$ a intensidade máxima (4) em seus 14 itens (Hamilton, 1959). Considera-se como remissão do TP a CGI $\leq 2$ e ausência de ataques de pânico (Heldt et al., 2003).

As escalas de autorrelato foram o Inventário de Depressão de Beck (BDI) (Gorenstein \& Andrade, 1998) e o Inventário de Temperamento e de Caráter (ITC) (Cloninger et al., 1993; Fuentes et al., 2000) . O BDI está validado para o português (Gorenstein \& Andrade, 1998), com o objetivo de identificar e quantificar quadros de depressão leve, moderada e grave tanto em pacientes internados quanto em pacientes ambulatoriais. Consiste de 21 itens, incluindo sintomas e atitudes cujas intensidades variam de 0 a 3 . Escores inferiores a 10 representam ausência de depressão; escores de 10 a 18, depressão leve; de 19 a 29, depressão moderada e, acima de 30, depressão grave. O ITC é um questionário de autopreenchimento composto por 240 itens do tipo "verdadeiro ou falso" (Cloninger et al., 1993), que foi validado para o português (Fuentes et al., 2000). A aplicação do ITC foi única no início da TCCG, e a versão traduzida obteve um índice geral de concordância com a versão original determinada pelo coeficiente de correlação intraclasse em todos os fatores de temperamento e caráter.

\section{Intervenção}

O protocolo padrão de TCCG utilizado foi adaptado do manual de Otto e colaboradores (Otto \& Deckersbach, 1998) e foi descrito em estudos prévios (Heldt et al., 2006; Heldt et al., 2003). Ao todo, foram realizados nove grupos entre os anos de 2006 e 2011, com sessões semanais de 90 minutos. Cada grupo teve duração de quatro meses e contou, em média, com sete pacientes participantes. Todos os grupos foram coordenados por profissionais (enfermeiras psiquiátricas, psicólogas e residentes em psiquiatria) com experiência prévia em TCCG, sendo que sete grupos apresentaram a mesma coordenação.

O protocolo de 12 sessões de TCCG para TP utilizou os seguintes recursos: psicoeducação, técnicas para enfrentamento da ansiedade, reestruturação cognitiva, exposição interoceptiva e naturalística e exposição ao vivo para a conduta evitativa (Heldt et al., 2006; Heldt et al., 2003). As primeiras sessões foram destinadas à compreensão cognitiva do medo e à aprendizagem de técnicas para enfrentar a ansiedade (relaxamento muscular e respiração diafragmática). A seguir, o foco recaiu sobre os pensamentos automáticos, etapa em que os pacientes identificavam e avaliavam as evidências das interpretações catastróficas. A simulação de sintomas físicos foi praticada em sessão e como tarefa de casa durante a fase intermediária do tratamento através da exposição interoceptiva (taquicardia, tontura, falta de ar e desrealização). A exposição naturalística foi conduzida em grupo, com as sensações evocadas de modo natural, como, por exemplo, ao subir escadas. Ao final do tratamento, iniciou-se a exposição in vivo de forma gradativa, isto é, os pacientes elaboravam uma lista hierarquizada das situações evitadas e as tarefas foram combinadas de maneira individualizada, iniciando-se pelo menor grau de dificuldade, e os avanços e dificuldades eram acompanhados e discutidos em sessão. As sessões seguintes caracterizaram-se pela revisão das exposições e pelo aumento do grau de dificuldade das situações.

Basicamente, cada sessão era organizada em três etapas: nos primeiros minutos era realizada conexão com a sessão anterior, principalmente com a revisão das tarefas combinadas. A seguir, ocorriam os procedimentos para atingir o objetivo proposto para a sessão. No encerramento, eram combinadas as tarefas a serem realizadas até a próxima sessão.

\section{Análise dos Dados}

A análise estatística do banco de dados foi realizada utilizando-se o Statistical Package for the Social Sciences (SPSS) versão 18.0 e o nível de significância adotado foi de $\alpha=0,05$. As mudanças na intensidade dos sintomas ao longo do tratamento foram avaliadas com o teste $t$ para medidas pareadas e o tamanho de efeito do tratamento foi calculado pela fórmula de Cohen $(E S=M 1-M 2 / \sqrt{ } S D 12+S D 22-2$ $\mathrm{x} r \mathrm{x} S D 1 \times S D 2)$.

Para verificar se as características de temperamento e caráter influenciaram a resposta à TCCG, foi utilizado o Generalized Estimating Equations (GEE), e a associação com comorbidades de eixo I foi analisada com o teste $t$ para amostra independente. Para identificar as variáveis independentes de resposta à TCCG, foi realizada a regressão linear entre as características de temperamento e caráter com $\mathrm{p}<0,05$ após a análise de GEE e a mudança da gravidade dos sintomas.

O tamanho amostral foi calculado considerando-se um poder de $90 \%$, alfa de 0,05 e desvio-padrão de 0,9 para um tamanho de efeito de 0,7 . Obteve-se uma amostra aproximada de 42 indivíduos.

\section{Resultados}

Um total de $55(87 \%)$ pacientes realizou a TCCG e as oito perdas foram devido à desistência por motivos pessoais (mudança de endereço, mudança de emprego e doença clínica; $n=3$ ) e por má adesão (faltaram mais que $50 \%$ das sessões; $\mathrm{n}=5$ ). Entre aqueles que concluíram o tratamento, 40 ( $73 \%$ ) eram do sexo feminino, com média de idade de 39 anos ( $D P=10,93$; mínimo 22 e máximo 65 anos), com 11 anos de estudo $(D P=3,92)$. Trinta e cinco participantes $(64 \%)$ tinham um companheiro. Tanto as características clínicas quanto as de temperamento e caráter estão apresentadas na Tabela 1. Nota-se longo tempo de doença mediana (10 anos; intervalo interquartis 3,5 a 17,5), predomínio de comorbidades de eixo I e uso de medicação. Devido à alta prevalência da 
comorbidade de agorafobia, em 45 (82\%) pacientes não foi considerado, para fins de análise, a agorafobia como outra doença de eixo I.

As comorbidades com transtorno de humor foram: episódio depressivo maior - 18 (33\%); distimia - 7 (13\%); e episódio hipomaníaco - $3(5 \%)$. As comorbidades com outros transtornos de ansiedade compreenderam: ansiedade social - 8 (14\%); transtorno obsessivo compulsivo - 5 (9\%); transtorno de estresse pós-traumático - $1(2 \%)$; e transtorno de ansiedade generalizada - 23 (42\%).

Observou-se que o uso de medicação antidepressiva não apresentou alteração significativa avaliada após a intervenção

Tabela 1. Características clínicas dos pacientes no início da $\operatorname{TCCG}(n=55)$

\begin{tabular}{|c|c|c|}
\hline Características & $\mathbf{N}(\%)$ & Média \pm DP \\
\hline Idade de início dos sintomas (anos) & & $26,87 \pm 11,85$ \\
\hline \multicolumn{3}{|l|}{ Comorbidades } \\
\hline Transtornos de humor ${ }^{\mathrm{a}}$ & $28(51)$ & \\
\hline Transtornos de ansiedade ${ }^{\mathrm{b}}$ & $28(51)$ & \\
\hline \multicolumn{3}{|l|}{ Medicações ${ }^{\mathrm{c}}$} \\
\hline Antidepressivos & $43(78)$ & \\
\hline Benzodiazepínicos & $30(54)$ & \\
\hline Mais de uma medicação & $27(49)$ & \\
\hline \multicolumn{3}{|l|}{$\begin{array}{r}\text { Inventário de Temperamento e } \\
\text { Caráter (ITC) }\end{array}$} \\
\hline Busca de novidade & & $18,84 \pm 5,66$ \\
\hline Esquiva ao dano & & $24,60 \pm 5,75$ \\
\hline Dependência de gratificação & & $15,47 \pm 4,25$ \\
\hline Persistência & & $4,35 \pm 2,11$ \\
\hline Autodirecionamento & & $25,84 \pm 7,66$ \\
\hline Cooperatividade & & $30,71 \pm 7,00$ \\
\hline Autotranscendência & & $17,65 \pm 6,28$ \\
\hline
\end{tabular}

Nota. Abreviaturas: TCCG: terapia cognitivo-comportamental em grupo; DP: desvio padrão

a Episódio depressivo maior, transtorno distímico, episódio hipomaníaco.

${ }^{\mathrm{b}}$ Ansiedade social, transtorno obsessivo compulsivo, transtorno de estresse pós-traumático, transtorno de ansiedade generalizada.

${ }^{c}$ Antidepressivo: inibidor seletivo de recaptação da serotonina e tricíclicos. (antes=43 e depois=39; $p=0,125)$. Entretanto, o uso de benzodiazepínicos diminuiu significativamente após a TCCG (antes $=30$ e depois $=12 ; p=0,003)$.

\section{Resposta à TCCG e Características de Temperamento e Caráter}

A TCCG foi efetiva quanto à melhora de todas as medidas de desfecho. Os resultados estão apresentados na Tabela 2. Não foi encontrada associação significativa com as características de temperamento e caráter, bem como com a resposta ao tratamento, considerando os sintomas do TP. Porém, houve associação significativa entre fatores de caráter e mudanças da agorafobia e da depressão (Tabela 3).

Menores escores de autodirecionamento apresentaram associação com melhora da agorafobia $(p=0,021)$ e dos sintomas depressivos $(p=0,049)$. Menores escores de persistência e de cooperatividade foram associados significativamente à melhora dos sintomas depressivos ( $p=0,019$ e 0,007 , respectivamente). Os demais fatores não apresentaram associação significativa.

Para verificar a relação entre as comorbidades de eixo I e as características de temperamento e caráter, foi realizada análise com o teste $t$ para amostras independentes e os resultados estão apresentados na Tabela 4. Observou-se que o escore do fator de temperamento esquiva ao dano foi significativamente maior $(p=0,015)$ e o escore do fator de caráter autodirecionamento foi menor $(p=0,050)$ na presença de comorbidade com outros transtornos de ansiedade. Não foi encontrado diferença significativa entre a comorbidade com transtorno de humor e a remissão do TP (CGI $\leq 2$ e sem ataques de pânico) com os fatores de temperamento e caráter.

Também foi realizada análise de regressão linear para verificar se a melhora da agorafobia e dos sintomas depressivos após a TCCG era influenciada pelas características de temperamento e caráter de modo independente. Constatou-se que a melhora foi devida à maior gravidade da agorafobia $(\beta=0,656 ; p<0,001)$ antes de iniciar a TCCG e o autodirecionamento não foi mais significativo $(\beta=0,155 ; p=0,186)$. Da mesma forma, a melhora dos sintomas depressivos foi relacionada a maiores escores do BDI antes do tratamento $(\beta=0,618 ; p<0,001)$, enquanto

Tabela 2. Resposta a Terapia Cognitivo-Comportamental em Grupo

\begin{tabular}{lccccc}
\hline \multirow{2}{*}{ Sintomas } & \multicolumn{2}{c}{ TCCG } & & \\
\cline { 2 - 3 } ICG & Antes & Depois & & TE \\
Agorafobia & $4,53 \pm 0,94$ & $2,82 \pm 1,07$ & $<0,001$ & 1,19 \\
Ataques de Pânico (intensidade) & $5,36 \pm 3,64$ & $2,80 \pm 3,38$ & $<0,001$ & 0,56 \\
Ansiedade Antecipatória & $7,94 \pm 2,59$ & $4,60 \pm 3,03$ & $<0,001$ & 1,00 \\
Ansiedade (HAM-A) & $29,62 \pm 8,66$ & $17,73 \pm 9,49$ & $<0,001$ & 1,47 \\
Depressão (BDI) & $19,02 \pm 13,28$ & $11,69 \pm 11,34$ & $<0,001$ & 0,66 \\
\hline
\end{tabular}

Nota. Teste T para amostras pareadas.

Abreviaturas: TCCG: terapia cognitivo-comportamental em grupo; ICG: Impressão

Clínica Global; HAM-A: Escala Hamilton de Ansiedade; BDI: Inventário de Depressão de

Beck; TE: tamanho de efeito 
o autodirecionamento $(\beta=0,011 ; p=0,930)$, a persistência $(\beta=0,176 ; \mathrm{p}=0,111)$ e a cooperatividade $(\beta=0,114 ; p=0,343)$ não foram mais significativos.

\section{Discussão}

Os resultados mostraram uma melhora significativa dos sintomas do TP e depressivos após tratamento, confirmando as evidências prévias da efetividade da TCCG (Heldt et al., 2003; Otto \& Deveney, 2005). O temperamento e caráter não influenciaram na resposta ao tratamento do TP. Entretanto, menor autodirecionamento, característico de pessoas que tendem a se responsabilizar menos por suas escolhas e sua individualidade, e maior esquiva ao dano, relacionado à inibição de comportamentos para enfrentar estímulos aversivos (Cloninger et al., 1993), estiveram associados à presença de comorbidade de TP com outros transtornos de ansiedade. De acordo com estudos prévios, baixo autodirecionamento e alta esquiva ao dano são característicos de pacientes com TP (Wachleski et al., 2008) e são frequentes em transtornos de ansiedade (Cloninger, Zohar, Hirschmann, \& Dahan, 2012; Rettew et al., 2006). Também, essas características apresentaram impacto negativo na resposta à TCC para o TOC (Corchs et al., 2008).
Embora na análise bivariada os menores escores da cooperatividade (interesse e tolerância com o meio) e da persistência (trata de insistir na resposta de determinada forma) relacionaram-se com a diminuição dos sintomas depressivos, e o autodirecionamento com a melhora da agorafobia, os resultados não se mantiveram significativos na análise de regressão linear. É provável que, devido aos pacientes apresentarem maior intensidade dos sintomas, houvesse maior possibilidade para a melhora. Logo, as características de temperamento e caráter não estiveram associadas à resposta ao tratamento com TCCG, quando controlada a gravidade inicial dos sintomas.

No modelo psicobiológico da personalidade proposto por Cloninger et al. (1993), o temperamento e o caráter influenciam-se mutuamente e motivam o comportamento. As respostas automáticas para iniciar, manter ou interromper uma ação são primeiramente determinadas por fatores de temperamento. No entanto, essas respostas podem ser modificadas em consequência de alterações no significado do estímulo, que é influenciado pelos fatores concernentes ao caráter.

Nesse sentido, compreende-se que o temperamento apresenta um substrato biológico e passível de mudança com tratamento farmacológico, o que não ocorre com o caráter (Cloninger et al., 1993; Marchesi et al., 2008). Os pacientes,

Tabela 3. Associação entre resposta a TCCG e os fatores de temperamento e caráter*

\begin{tabular}{|c|c|c|c|c|c|c|}
\hline \multirow{3}{*}{$\begin{array}{c}\text { Inventário de temperamento e } \\
\text { caráter }\end{array}$} & \multirow[b]{2}{*}{ ICG } & \multirow[b]{2}{*}{$\begin{array}{l}\text { Ansiedade } \\
\text { (HAM-A) }\end{array}$} & \multicolumn{3}{|c|}{ Inventário do Pânico } & \multirow[b]{2}{*}{$\begin{array}{c}\text { Depressão } \\
\text { (DBI) }\end{array}$} \\
\hline & & & Agorafobia & $\begin{array}{c}\text { Ansiedade } \\
\text { Antecipatória }\end{array}$ & Ataques & \\
\hline & $B$ & $B$ & $B$ & $B$ & $B$ & $B$ \\
\hline Busca de novidades & $-0,021$ & $-0,113$ & 0,011 & 0,038 & $-3,154$ & 0,324 \\
\hline Esquiva ao dano & $-0,023$ & 0,036 & 0,083 & $-0,053$ & 0,004 & 0,229 \\
\hline Dependência de gratificação & 0,032 & 0,166 & 0,035 & 0,147 & 0,013 & $-0,477$ \\
\hline Persistência & $-0,081$ & $-0,657$ & $-0,298$ & $-0,068$ & $-0,072$ & $-1,378^{*}$ \\
\hline Autodirecionamento & 0,021 & $-0,166$ & $-0,124 *$ & $-0,026$ & $-0,009$ & $-0,392 *$ \\
\hline Cooperatividade & 0,029 & $-0,013$ & $-0,069$ & 0,032 & 0,008 & $-0,528^{*}$ \\
\hline Autotranscendência & 0,028 & $-0,02$ & 0,018 & 0,061 & $-0,015$ & $-0,169$ \\
\hline
\end{tabular}

Nota. GEE $=$ Generalized Estimating Equations; $\mathrm{B}=$ Interação entre sintoma e fator; ${ }^{*} p<0,05 ;{ }^{*} p<0,001$.

Abreviaturas: ICG: Impressão Clínica Global; HAM-A: Escala Hamilton de Ansiedade; BDI: Inventário de Depressão de Beck.

Tabela 4. Comparação entre comorbidades e remissão do TP com os fatores de temperamento e caráter

\begin{tabular}{lcccccc}
\hline \multicolumn{1}{c}{$\begin{array}{c}\text { Fatores de temperamento e } \\
\text { caráter }\end{array}$} & \multicolumn{2}{c}{ Transtorno de humor } & \multicolumn{2}{c}{ Transtornos de ansiedade $^{\mathbf{b}}$} & \multicolumn{2}{c}{ Remissão $^{\mathbf{c}}$} \\
\cline { 2 - 7 } & Presente & Ausente & Presente & Ausente & Presente & Ausente \\
\hline Busca de novidades & $19,54 \pm 5,61$ & $18,11 \pm 5,72$ & $19,21 \pm 5,29$ & $18,44 \pm 6,10$ & $18,80 \pm 4,24$ & $18,86 \pm 6,39$ \\
Esquiva ao dano & $25,96 \pm 4,76$ & $23,19 \pm 6,40$ & $26,43 \pm 5,10^{*}$ & $22,70 \pm 5,85^{*}$ & $23,20 \pm 4,68$ & $25,40 \pm 6,2$ \\
Dependência de gratificação & $15,54 \pm 4,52$ & $15,41 \pm 4,03$ & $14,86 \pm 4,56$ & $16,11 \pm 3,88$ & $16,15 \pm 3,73$ & $15,09 \pm 4,52$ \\
Persistência & $4,04 \pm 2,10$ & $4,67 \pm 2,11$ & $4,39 \pm 2,20$ & $4,30 \pm 2,05$ & $4,05 \pm 1,70$ & $4,51 \pm 2,31$ \\
Autodirecionamento & $24,29 \pm 7,30$ & $27,44 \pm 7,84$ & $23,86 \pm 7,71^{*}$ & $27,89 \pm 7,19 *$ & $26,80 \pm 7,91$ & $25,29 \pm 7,58$ \\
Cooperatividade & $30,93 \pm 7,18$ & $30,48 \pm 6,94$ & $29,29 \pm 8,11$ & $32,19 \pm 5,39$ & $32,25 \pm 6,44$ & $29,83 \pm 7,25$ \\
Autotranscendência & $18,86 \pm 7,36$ & $16,41 \pm 4,75$ & $17,89 \pm 5,63$ & $17,41 \pm 6,99$ & $17,10 \pm 6,63$ & $17,97 \pm 6,15$ \\
\hline
\end{tabular}

Nota. Teste $T$ para amostras independentes; * $p<0,05$.

a Transtorno de humor - depressão atual e passada, distimia, episódio hipomaníaco (vida inteira e atual).

b Transtornos de ansiedade - com sintomas atuais de fobia social, transtorno obsessivo-compulsivo, transtorno de estresse pós-traumático, transtorno de ansiedade generalizada.

${ }^{c}$ Critério de remissão: $\mathrm{ICG} \leq 2$ e sem ataques de pânico. 
ao ingressarem na TCCG, utilizavam medicações de modo que esse aspecto foi controlado no estudo. A abordagem utilizada foi psicológica, agindo nos aspectos cognitivos e na expressão do comportamento, ou seja, os pacientes passam a significar estímulos internos e externos com novas alternativas de comportamento. Assim, é possível que as características menos intensas de caráter exerçam influência na intensidade dos sintomas, mas não na resposta ao tratamento. Os nossos resultados estão de acordo com outro estudo que apontou para a ausência de restrição quanto à indicação de TCC para pessoas com diferentes características de personalidade (Telch et al., 2011).

Este estudo apresenta limitações devido à ausência de grupo controle e ao pequeno tamanho da amostra. A avaliação da personalidade também apresenta variações nos construtos conforme os distintos instrumentos utilizados, o que pode dificultar a comparação entre os estudos. Outras limitações são as variáveis vinculadas aos terapeutas e características específicas de cada um dos nove grupos que não foram controladas nas análises. Ainda, um aspecto a ser considerado é relativo à adesão ao tratamento, o qual pode repercutir na resposta à TCCG e não foi avaliado no estudo. Contudo, trata-se de uma pesquisa com repercussões clínicas e novos estudos que incluam avaliação de diagnósticos de personalidade em pacientes com TP e resposta a TCCG ainda precisam ser desenvolvidos.

\section{Referências}

Amorim, P. (2000). Mini International Neuropsychiatric Interview (MINI): Validation of a short structured diagnostic psychiatric interview. Revista Brasileira de Psiquiatria, 22, 106-155.

Bienvenu, O. J., Stein, M. B., Samuels, J. F., Onyike, C. U., Eaton, W. W., \& Nestadt, G. (2009). Personality disorder traits as predictors of subsequent first-onset panic disorder or agoraphobia. Comprehensive Psychiatry, 50(3), 209-214. doi: 10.1016/j.comppsych.2008.08.006

Bulik, C. M., Sullivan, P. F., Carter, F. A., McIntosh, V. V., \& Joyce, P. R. (1999). Predictors of rapid and sustained response to cognitive-behavioral therapy for bulimia nervosa. International Journal of Eating Disorders, 26(2), 137-144.

Chavira, D. A., Stein, M. B., Golinelli, D., Sherbourne, C. D., Craske, M. G., Sullivan, G., ... Roy-Byrne, P. P. (2009). Predictors of clinical improvement in a randomized effectiveness trial for primary care patients with panic disorder. The Journal of Nervous and Mental Disease, 197(10), 715-721. doi: 10.1097/ NMD.0b013e3181b97d4d

Cloninger, C. R., Svrakic, D. M., \& Przybeck, T. R. (1993). A psychobiological model of temperament and character. Archives of General Psychiatry, 50(12), 975-990.

Cloninger, C. R., Zohar, A. H., Hirschmann, S., \& Dahan, D. (2012). The psychological costs and benefits of being highly persistent: Personality profiles distinguish mood disorders from anxiety disorders. Journal of Affective Disorders, 136(3), 758-766. doi: 10.1016/j.jad.2011.09.046

Corchs, F., Corregiari, F., Ferrao, Y. A., Takakura, T., Mathis, M. E., Lopes, A. C., ... Bernik, M. (2008). Personality traits and treatment outcome in obsessive-compulsive disorder. Revista Brasileira de Psiquiatria, 30(3), 246-250.
Fuentes, D., Tavares, H., Camargo, C. H. P., \& Gorenstein, C. (2000). Inventário de Temperamento e de Caráter de Cloninger - Validação da Versão em Português. In C. Gorenstein, L. H. S. G. Andrade, \& A. W. Zuardi (Eds.), Escalas de avaliação clínica em psiquiatria e psicofarmacologia (pp. 363-369). São Paulo: Lemos.

Gorenstein, C., \& Andrade, L. H. (1998). Inventário de Depressão de Beck: Propriedades psicométricas da versão em português. Revista de Psiquiatria Clínica, 25, 245-250.

Guy, W. (1979). CGI: Clinical Global Impressions. In W. Guy (Ed.), ECDEU Assessment manual for psychopharmacology (pp. 217-222). Rockville, MD, U.S. Department of Health, Education, and Welfare.

Hamilton, M. (1959). The assessment of anxiety states by rating. British Journal of Medical Psychology, 32(1), 50-55.

Heldt, E., Gus Manfro, G., Kipper, L., Blaya, C., Isolan, L., \& Otto, M. W. (2006). One-year follow-up of pharmacotherapyresistant patients with panic disorder treated with cognitivebehavior therapy: Outcome and predictors of remission. Behaviour Research Therapy, 44(5), 657-665. doi: 10.1016/j. brat.2005.05.003

Heldt, E., Kipper, L., Blaya, C., Salum, G. A., Hirakata, V. N., Otto, M. W., \& Manfro, G. G. (2011). Predictors of relapse in the second follow-up year post cognitive-behavior therapy for panic disorder. Revista Brasileira de Psiquiatria, 33(1), 23-29.

Heldt, E., Manfro, G. G., Kipper, L., Blaya, C., Maltz, S., Isolan, L., ... Otto, M. W. (2003). Treating medication-resistant panic disorder: predictors and outcome of cognitive-behavior therapy in a Brazilian public hospital. Psychotherapy and Psychosomatics, 72(1), 43-48. doi: 67188

Joyce, P. R., McKenzie, J. M., Carter, J. D., Rae, A. M., Luty, S. E., Frampton, C. M., \& Mulder, R. T. (2007). Temperament, character and personality disorders as predictors of response to interpersonal psychotherapy and cognitive-behavioural therapy for depression. British Journal of Psychiatry, 190, 503-508. doi: 10.1192/bjp.bp.106.024737

Kipper, L., Wachleski, C., Salum, G. A., Heldt, E., Blaya, C., \& Manfro, G. G. (2009). Can psychopharmacological treatment change personality traits in patients with panic disorder? Revista Brasileira de Psiquiatria, 31(4), 307-313.

Latas, M., \& Milovanovic, S. (2014). Personality disorders and anxiety disorders: What is the relationship? Current Opinion of Psychiatry, 27(1), 57-61. doi: 10.1097/ YCO.0000000000000025

Marchesi, C., Cantoni, A., Fonto, S., Giannelli, M. R., \& Maggini, C. (2006). The effect of temperament and character on response to selective serotonin reuptake inhibitors in panic disorder. Acta Psychiatrica Scandinavica, 114(3), 203-210. doi: 10.1111/j.1600-0447.2006.00772.x

Marchesi, C., De Panfilis, C., Cantoni, A., Giannelli, M. R., \& Maggini, C. (2008). Effect of pharmacological treatment on temperament and character in panic disorder. Psychiatry Research, 158(2), 147-154. doi: 10.1016/j. psychres.2006.08.009

Mendoza, L., Navines, R., Crippa, J. A., Fagundo, A. B., Gutierrez, F., Nardi, A. E., ... Martin-Santos, R. (2011). Depersonalization and personality in panic disorder. Comprehensive Psychiatry, 52(4), 413-419. doi: 10.1016/j.comppsych.2010.09.002 
Mitte, K. (2005). A meta-analysis of the efficacy of psychoand pharmacotherapy in panic disorder with and without agoraphobia. Journal of Affective Disorders, 88(1), 27-45. doi: 10.1016/j.jad.2005.05.003

Otto, M. W., \& Deckersbach, T. (1998). Cognitive behavioral therapy for panic disorder. In J. R. Rosenbaum \& M. H. Pollack (Eds.), Panic disorder and its treatment (pp. 181-203). New York: Marcel Decker.

Otto, M. W., \& Deveney, C. (2005). Cognitive-behavioral therapy and the treatment of panic disorder: Efficacy and strategies. Journal of Clinical Psychiatry, 66 Suppl 4, 28-32.

Pollack, M. H. (2005). The pharmacotherapy of panic disorder. Journal of Clinical Psychiatry, 66 Suppl 4, 23-27.

Prasko, J., Houbova, P., Novak, T., Zalesky, R., Espa-Cervena, K., Paskova, B., \& Vyskocilova, J. (2005). Influence of personality disorder on the treatment of panic disorder--comparison study. Neuro Endocrinology Letters, 26(6), 667-674.
Rettew, D. C., Doyle, A. C., Kwan, M., Stanger, C., \& Hudziak, J. J. (2006). Exploring the boundary between temperament and generalized anxiety disorder: a receiver operating characteristic analysis. Journal of Anxiety Disorders, 20(7), 931-945. doi: 10.1016/j.janxdis.2006.02.002

Skodol, A. E., Geier, T., Grant, B. F., \& Hasin, D. S. (2014). Personality disorders and the persistence of anxiety disorders in a nationally representative sample. Depression and Anxiety, 31(9), 721-728. doi: 10.1002/da.22287

Telch, M. J., Kamphuis, J. H., \& Schmidt, N. B. (2011). The effects of comorbid personality disorders on cognitive behavioral treatment for panic disorder. Journal Psychiatric Research, 45(4), 469-474. doi: 10.1016/j.jpsychires.2010.08.008

Wachleski, C., Salum, G. A., Blaya, C., Kipper, L., Paludo, A., Salgado, A. P., \& Manfro, G. G. (2008). Harm avoidance and self-directedness as essential features of panic disorder patients. Comprehensive Psychiatry, 49(5), 476-481. doi: 10.1016/j. comppsych.2008.03.003 